\title{
Common Fixed Points of Two Mappings regarding a Generalized $c$-Distance over a Banach Algebra
}

\author{
Ladan Aryanpour (D), Hamidreza Rahimi (D), and Ghasem Soleimani Rad \\ Department of Mathematics, Faculty of Science, Central Tehran Branch, Islamic Azad University, Tehran, Iran \\ Correspondence should be addressed to Hamidreza Rahimi; rahimi@iauctb.ac.ir
}

Received 25 October 2020; Revised 30 January 2021; Accepted 18 February 2021; Published 11 March 2021

Academic Editor: Huseyin Isik

Copyright (c) 2021 Ladan Aryanpour et al. This is an open access article distributed under the Creative Commons Attribution License, which permits unrestricted use, distribution, and reproduction in any medium, provided the original work is properly cited.

\begin{abstract}
In this article, applying the concept of a generalized $c$-distance in cone $b$-metric spaces over Banach algebra with a nonnormal solid cone therein, we establish several common fixed point theorems for two noncontinuous mappings satisfying the Han-Xu-type contraction. Our results are interesting, since they are not equivalent to former well-known results regarding a $w t$-distance in $b$ -metric spaces while they contain recent results corresponding to a generalized $c$-distance in cone $b$-metric spaces.
\end{abstract}

\section{Introduction and Preliminaries}

In 2015, Bao et al. [1] suggested a generalized $c$-distance, which extended many former definitions in [2-10]) and references therein. Moreover, with regard to a survey on fixed point theory corresponding to this distance, see [11-13]).

In 2013, Liu and $\mathrm{Xu}$ [14] offered a cone metric space over Banach algebra by replacing a Banach space $E$ with a Banach algebra $\mathscr{E}$. After this definition, some other researchers suggested new several various spaces over a Banach algebra and extended available results in [15-17] and their references. In 2015, Huang et al. [18] proposed a $c$-distance in cone metric spaces over a Banach algebra $\mathscr{E}$. In 2018, Han and $\mathrm{Xu}$ [19] proved some common fixed point results by removing the assumption of continuity of the mappings and by deleting the hypothesis of the normality of the cone. Recently, Arabnia et al. [20] suggested a generalized $c$-distance in cone $b$-metric spaces over a Banach algebra.

Here, we review some basic definitions and preliminary lemmas which are needed to continue.

Let $\mathscr{E}$ be a Banach algebra with a unit element $e$, a zero element $\theta$, a norm $\|x\|$, and a cone $P$ therein. Define a partial order $\leqslant$ with respect to $P$ by $x \leqslant y$ iff $y-x \in P$. Also, $x \leqslant y$ if $x \preccurlyeq y$ and $x \neq y$, and $x \ll y$ iff $y-x \in$ int $P$ (int $P$ is the same as the interior of $P$ ). If int $P \neq \varnothing$, then the cone $P$ is named solid. Further, if there is $M>0$ so that $\theta \preccurlyeq x \preccurlyeq y$ deduced that $\|x\| \leq M\|y\|$ for every $x, y \in \mathscr{E}$, then the cone $P$ is named a normal cone.

Definition 1 (see [17]). Let $\mathfrak{X}$ be a nonempty set, $s \geq 1$ be a constant, and $\mathscr{E}$ be a Banach algebra. For all $x, y, z \in \mathfrak{X}$, assume that $d: \mathfrak{X} \times \mathfrak{X} \rightarrow \mathscr{E}$ satisfies the following items: 2

$$
\begin{aligned}
& \left(\mathrm{d}_{1}\right) \theta \leqslant \mathrm{d}(x, y) \text { and } \mathrm{d}(x, y)=\theta \text { iff } x=y \\
& \left(\mathrm{~d}_{2}\right) \mathrm{d}(x, y)=\mathrm{d}(y, x) \\
& \left(\mathrm{d}_{3}\right) \mathrm{d}(x, z) \leqslant s[\mathrm{~d}(x, y)+\mathrm{d}(y, z)]
\end{aligned}
$$

Then, $\mathrm{d}$ is named a cone $b$-metric on $\mathfrak{X}$, and $(\mathfrak{X}, \mathrm{d})$ is named a cone $b$-metric space over the Banach algebra $\mathscr{E}$.

For definitions such as convergent and Cauchy sequences, $c$-sequence, completeness, continuity, and examples, see [16, 17]. In the sequel, assume that $(\mathfrak{X}, \mathrm{d})$ is a cone $b$-metric space with the coefficient $s \geq 1$ over a Banach algebra $\mathscr{E}$ and $P$ is a solid cone therein.

Lemma 2 (see $[17,21]$ ). Let $\mathscr{E}$ be a Banach algebra and $u, v$ $\in \mathscr{E}$. Then, the following items hold:

$\left(l_{1}\right)$ if $\rho(u)<|w|$ where $\rho(u)$ is the spectral radius and $w$ is a complex constant, then $w e-u$ is invertible in $\mathscr{E}$ and

$$
(w e-u)^{-1}=\sum_{i=0}^{\infty} \frac{u^{i}}{w^{i+1}}, \rho\left((w e-u)^{-1}\right) \leq \frac{1}{|w|-\rho(u)}
$$


$\left(l_{2}\right)$ if $u$ commutes with $v$, then $\rho(u+v) \leq \rho(u)+\rho(v)$ and $\rho(u v) \leq \rho(u) \rho(v)$ $u=\theta$

$\left(l_{3}\right)$ if $u, k_{1}, k_{2} \in P$ with $k_{1} \preccurlyeq k_{2}, u \preccurlyeq k_{1} u$, and $\rho\left(k_{2}\right)<1$, then

$\left(l_{4}\right)$ if $\rho(u)<1$, then $\left\{u^{n}\right\}$ is a c-sequence. Furthermore, $\left\{k u^{n}\right\}$ is a c-sequence for each arbitrarily vector $k \in P$

Definition 3 (see [20]). Let $(\mathfrak{X}, d)$ be a cone $b$-metric space over a Banach algebra $\mathscr{E}$ with a constant $s \geq 1$. A function $v$ $: \mathfrak{X} \times \mathfrak{X} \rightarrow \mathscr{E}$ is named a generalized $c$-distance on $\mathfrak{X}$ if, for all $x, y, z \in \mathfrak{X}$, it satisfies in the following properties:

$\left(v_{1}\right) \theta \leqslant v(x, y)$

$\left(v_{2}\right) v(x, y) \leqslant s[v(x, z)+v(z, y)]$

$\left(v_{3}\right)$ for $x \in \mathfrak{X}$ and a sequence $\left\{y_{n}\right\}$ converges to $y$ in $\mathfrak{X}$, if $v\left(x, y_{n}\right) \preccurlyeq u$ for some $u=u_{x} \in P$ and all $n \geq 1$, then $v(x, y) \leqslant s u$

$\left(v_{4}\right)$ for all $c \in \mathscr{E}$ with $\theta \ll c$, there exists $e \in \mathscr{E}$ with $\theta \ll e$ so that $v(z, x) \ll e$ and $v(z, y) \ll e$ imply $\mathrm{d}(x, y) \ll c$

Notice that a generalized $c$-distance contains both $w t$ -distance and $c$-distance. Further, $v(x, y)=v(y, x)$ is not necessarily true and the $v(x, y)=\theta$ dose not imply that $x=y$ for every $x, y \in \mathfrak{X}$.

Example 4. Take $\mathfrak{X}=[0,1], \mathscr{E}=C_{\mathbb{R}}^{1}[0,1]$ with $\|f\|=\|f\|_{\infty}+\|$ $f^{\prime} \|_{\infty}$. Let multiplication in $\mathscr{E}$ be just pointwise multiplication. Then, $\mathscr{E}$ is a Banach algebra with a unit $e(t)=1$ for all $t \in[0,1]$. Also, let $P=\{f \in \mathscr{A} \mid f(t) \geq 0, \forall t \in[0,1]\}$ be a solid cone. Now, define $d: X \times X \rightarrow P \subset \mathscr{A}$ by $d(a, b)(t)=$ $|a-b|^{2} 2^{t}$ for all $a, b \in \mathfrak{X}$, where $2^{t} \in P$. Then, $(X, d)$ is a cone $b$-metric space over a Banach algebra $\mathscr{E}$. Consider a mapping $v: \mathfrak{X} \times \mathfrak{X} \rightarrow \mathscr{E}$ by $v(a, b)(t)=\left(a^{2}+b^{2}\right) 2^{t}$ for all $a, b \in \mathfrak{X}$. Then, $v$ is a generalized $c$-distance on $\mathfrak{X}$.

Lemma 5 (see [20]). Consider a generalized c-distance $v$ on $\mathfrak{X}$ with two sequences $\left\{t_{n}\right\}$ and $\left\{s_{n}\right\}$ in $\mathfrak{X}, \alpha, \beta, \gamma \in \mathfrak{X}$, and $\left\{p_{n}\right\}$ and $\left\{q_{n}\right\}$ be two c-sequences. Then, the following cases hold:

(1) if $v\left(t_{n}, \beta\right) \leqslant p_{n}$ and $v\left(t_{n}, \gamma\right) \leqslant q_{n}$ for $n \in \mathbb{N}$, then $\beta=\gamma$. In particular, if $\nu(\alpha, \beta)=\theta$ and $\nu(\alpha, \gamma)=\theta$, then $\beta=\gamma$

(2) if $v\left(t_{n}, s_{n}\right) \leqslant p_{n}$ and $v\left(t_{n}, \gamma\right) \preccurlyeq q_{n}$ for $n \in \mathbb{N}$, then $\left\{s_{n}\right\}$ converges to $\gamma$

(3) if $v\left(t_{n}, t_{m}\right) \leqslant p_{n}$ for $m>n$, then $\left\{t_{n}\right\}$ is a Cauchy sequence in $\mathfrak{X}$

(4) if $v\left(\beta, t_{n}\right) \leqslant p_{n}$ for $n \in \mathbb{N}$, then $\left\{t_{n}\right\}$ is a Cauchy sequence in $\mathfrak{X}$

Lemma 6 (see [20]). Let $v$ be a generalized c-distance on $\mathfrak{X}$. If $v(\alpha, \beta)=v(\beta, \alpha)=\theta$ for $\alpha, \beta \in \mathfrak{X}$, then $\alpha=\beta$.

In this work, we establish several common fixed point theorems regarding a generalized $c$-distance over a Banach algebra by removing the normality of the cone and the continuity of the mappings.

\section{Main Results}

The following theorem is the principal result of this paper using Han-Xu-type contraction [19].

Theorem 7. Consider a generalized $c$-distance $v$ on a complete cone b-metric space $(\mathfrak{X}, d)$ over a Banach algebra $\mathscr{E}$. Assume that two mappings $F, G: \mathfrak{X} \rightarrow \mathfrak{X}$ for every $a, b \in \mathfrak{X}$ satisfy the following relations:

$$
\begin{gathered}
v(F a, G b) \leqslant h_{1} v(a, b)+h_{2} v(a, F a)+h_{3} v(a, G b), \\
v(G a, F b) \leqslant h_{1} v(a, b)+h_{2} v(a, G a)+h_{3} v(a, F b),
\end{gathered}
$$

where $h_{1}, h_{2}, h_{3} \in P$ so that $h_{3}$ commutes with $\left(h_{1}+h_{2}\right.$ $\left.+s h_{3}\right)$ and

$$
\rho\left(s h_{3}\right)+\rho\left(s h_{1}+s h_{2}+s^{2} h_{3}\right)<1 \text {. }
$$

Then, $F$ and $G$ have a unique common fixed point.

Proof. Assume that $a_{0} \in \mathfrak{X}$ is an arbitrary point with $F a_{0} \neq a_{0}$. Consider the sequence $\left\{a_{n}\right\}$ by putting $a_{2 n+1}=F a_{2 n}$ and $a_{2 n+2}=G a_{2 n+1}$ for all $n \in \mathbb{N}$. Applying relation (2) by $a=a_{2 n}$ and $b=a_{2 n+1}$, we get

$$
\begin{aligned}
v\left(a_{2 n+1}, a_{2 n+2}\right)= & v\left(F a_{2 n}, G a_{2 n+1}\right) \leqslant h_{1} v\left(a_{2 n}, a_{2 n+1}\right) \\
& +h_{2} v\left(a_{2 n}, F a_{2 n}\right)+h_{3} v\left(a_{2 n}, G a_{2 n+1}\right) \\
\leqslant & h_{1} v\left(a_{2 n}, a_{2 n+1}\right)+h_{2} v\left(a_{2 n}, a_{2 n+1}\right) \\
& +\operatorname{sh}_{3}\left[v\left(a_{2 n}, a_{2 n+1}\right)+v\left(a_{2 n+1}, a_{2 n+2}\right)\right],
\end{aligned}
$$

for all $n \in \mathbb{N}$, which induces that

$$
\left(e-s h_{3}\right) v\left(a_{2 n+1}, a_{2 n+2}\right) \preccurlyeq\left(h_{1}+h_{2}+s h_{3}\right) v\left(a_{2 n}, a_{2 n+1}\right) \text {. }
$$

Similarly, applying relation (3) by $a=a_{2 n+1}$ and $b=a_{2 n+2}$, we get

$$
\begin{aligned}
v\left(a_{2 n+2}, a_{2 n+3}\right)= & v\left(G a_{2 n+1}, F a_{2 n+2}\right) \\
\leqslant & h_{1} v\left(a_{2 n+1}, a_{2 n+2}\right)+h_{2} v\left(a_{2 n+1}, a_{2 n+2}\right) \\
& +s_{3}\left[v\left(a_{2 n+1}, a_{2 n+2}\right)+v\left(a_{2 n+2}, a_{2 n+3}\right)\right]
\end{aligned}
$$

for all $n \in \mathbb{N}$, which induces that

$$
\left(e-s h_{3}\right) v\left(a_{2 n+2}, a_{2 n+3}\right) \preccurlyeq\left(h_{1}+h_{2}+s h_{3}\right) v\left(a_{2 n+1}, a_{2 n+2}\right) \text {. }
$$

Now, the inequalities (6) and (8) show that

$$
\left(e-s h_{3}\right) v\left(a_{n}, a_{n+1}\right) \preccurlyeq\left(h_{1}+h_{2}+s h_{3}\right) v\left(a_{n-1}, a_{n}\right) \text {. }
$$

Since $\rho\left(s h_{3}\right)<1$ (by relation (4)), it follows from Lemma $2,\left(l_{1}\right)$, that $\left(e-s h_{3}\right)$ is invertible and $\left(e-s h_{3}\right)^{-1}=\sum_{i=0}^{\infty}\left(s h_{3}\right)^{i}$. Let $h=\left(e-s h_{3}\right)^{-1}\left(h_{1}+h_{2}+s h_{3}\right)$. Since $s h_{3}$ commutes with $h_{1}+h_{2}+s h_{3}$, we obtain 


$$
\left(e-s h_{3}\right)^{-1}\left(h_{1}+h_{2}+s h_{3}\right)=\left(h_{1}+h_{2}+s h_{3}\right)\left(e-s h_{3}\right)^{-1} .
$$

Now, set $h=\left(e-s h_{3}\right)^{-1}\left(h_{1}+h_{2}+s h_{3}\right)$. Then, by Lemma 2, $\left(l_{1}\right)$ and $\left(l_{2}\right)$, we obtain

$$
\begin{aligned}
\rho(h) & =\rho\left(\left(e-s h_{3}\right)^{-1}\left(h_{1}+h_{2}+s h_{3}\right)\right) \\
& \leq \frac{1}{1-\rho\left(s h_{3}\right)} \rho\left(h_{1}+h_{2}+s h_{3}\right)<\frac{1}{s},
\end{aligned}
$$

which implies that $(e-s h)^{-1}=\sum_{i=0}^{\infty}(s h)^{i}$. Moreover, by multiplying $\left(e-s h_{3}\right)^{-1}$ in relation (9), we get

$$
\begin{aligned}
v\left(a_{n}, a_{n+1}\right) & \preccurlyeq\left(e-s h_{3}\right)^{-1}\left(h_{1}+h_{2}+s h_{3}\right) v\left(a_{n-1}, a_{n}\right) \\
& =h v\left(a_{n-1}, a_{n}\right) \leqslant \cdots \leqslant h^{n} v\left(a_{0}, a_{1}\right) .
\end{aligned}
$$

Consider $m, n \in \mathbb{N}$ with $m>n \geq 1$. Using relation (12) and $\left(v_{2}\right)$, we deduce by a simple computation that

$$
v\left(a_{n}, a_{m}\right) \leqslant(e-s h)^{-1} \operatorname{sh}^{n} v\left(a_{0}, a_{1}\right) .
$$

Since $\rho(h)<1 / s$ and $s \geq 1$, we have $\rho(h)<1$ which means that $\left\{h^{n}\right\}$ is a $c$-sequence by Lemma $2,\left(1_{4}\right)$. Using Lemma 5 , (3), $\left\{a_{n}\right\}$ is a Cauchy sequence. Due to the completeness of the space $\mathfrak{X}$, there is a $u \in \mathfrak{X}$ so that $a_{n} \rightarrow u$ as $n \rightarrow \infty$. Using relation (13) and $\left(v_{3}\right)$, we have

$$
v\left(a_{n}, u\right) \preccurlyeq(e-s h)^{-1} s^{2} h^{n} v\left(a_{0}, a_{1}\right),
$$

which shows that

$$
\begin{aligned}
& v\left(a_{2 n+1}, u\right) \preccurlyeq(e-s h)^{-1} s^{2} h^{2 n+1} v\left(a_{0}, a_{1}\right), \\
& v\left(a_{2 n}, u\right) \leqslant(e-s h)^{-1} s^{2} h^{2 n} v\left(a_{0}, a_{1}\right) .
\end{aligned}
$$

Now, we establish that $F u=G u=u$. In relation (2), set $a=a_{2 n}$ and $b=u$. Then, we get

$$
\begin{aligned}
v\left(a_{2 n+1}, G u\right)= & v\left(F a_{2 n}, G u\right) \leqslant h_{1} v\left(a_{2 n}, u\right)+h_{2} v\left(a_{2 n}, a_{2 n+1}\right) \\
& +s h_{3}\left[v\left(a_{2 n}, a_{2 n+1}\right)+v\left(a_{2 n+1}, G u\right)\right],
\end{aligned}
$$

which induces that $\left(e-s h_{3}\right) v\left(a_{2 n+1}, G u\right) \leqslant h_{1} v\left(a_{2 n}, u\right)+$ $\left(h_{2}+s h_{3}\right) v\left(a_{2 n}, a_{2 n+1}\right)$. Note that $e-s h_{3}$ is invertible. Thus, by the inequalities (12) and (16), we get

$$
\begin{aligned}
v\left(a_{2 n+1}, G u\right) & \leqslant\left(e-s h_{3}\right)^{-1}\left[h_{1} v\left(a_{2 n}, u\right)+\left(h_{2}+s h_{3}\right) v\left(a_{2 n}, a_{2 n+1}\right)\right] \\
& \preccurlyeq\left(e-s h_{3}\right)^{-1}\left[h_{1}(e-s h)^{-1} s^{2} h^{2 n} v\left(a_{0}, a_{1}\right)+\left(h_{2}+s h_{3}\right) s h^{2 n} v\left(a_{0}, a_{1}\right)\right] \\
& =\left(e-s h_{3}\right)^{-1}\left[h_{1}(e-s h)^{-1} s^{2}+s\left(h_{2}+s h_{3}\right)\right] h^{2 n} v\left(a_{0}, a_{1}\right) .
\end{aligned}
$$

By considering the inequalities (15) and (18), Lemma 2, $\left(l_{4}\right)$, and Lemma 5, (1), we conclude that $G u=u$. Now, in relation (3), set $a=a_{2 n+1}$ and $b=u$. Then, we get

$$
\begin{aligned}
v\left(a_{2 n+2}, F u\right)= & v\left(G a_{2 n+1}, F u\right) \leqslant h_{1} v\left(a_{2 n+1}, u\right) \\
& +h_{2} v\left(a_{2 n+1}, a_{2 n+2}\right) \\
& +s h_{3}\left[v\left(a_{2 n+1}, a_{2 n+2}\right)+v\left(a_{2 n+2}, F u\right)\right],
\end{aligned}
$$

which induces that $\left(e-s h_{3}\right) v\left(a_{2 n+2}, F u\right) \leqslant h_{1} v\left(a_{2 n+1}, u\right)$ $+\left(h_{2}+s h_{3}\right) v\left(a_{2 n+1}, a_{2 n+2}\right)$. Note that $e-s h_{3}$ is invertible. Thus, by the inequalities (12) and (15), we get

$$
\begin{aligned}
v\left(a_{2 n+2}, F u\right) \leqslant & \left(e-s h_{3}\right)^{-1}\left[h_{1} v\left(a_{2 n+1}, u\right)\right. \\
& \left.+\left(h_{2}+s h_{3}\right) v\left(a_{2 n+1}, a_{2 n+2}\right)\right] \\
\preccurlyeq & \left(e-s h_{3}\right)^{-1}\left[h_{1}(e-s h)^{-1} s^{2} h^{2 n+1} v\left(a_{0}, a_{1}\right)\right. \\
& \left.+\left(h_{2}+s h_{3}\right) s h^{2 n+1} v\left(a_{0}, a_{1}\right)\right] \\
= & \left(e-s h_{3}\right)^{-1}\left[h_{1}(e-s h)^{-1} s^{2}\right. \\
& \left.+s\left(h_{2}+s h_{3}\right)\right] h^{2 n+1} v\left(a_{0}, a_{1}\right) .
\end{aligned}
$$

By considering the inequalities (16) and (20), Lemma 2, $\left(\mathrm{l}_{4}\right)$, and Lemma 5, (1), we conclude that $F u=u$. Consequently, $F u=G u=u$; that is, $u$ is a common fixed point of $F$ and $G$. Also, by using the relation (2), we have

$$
\begin{aligned}
v(u, u)= & v(F u, G u) \leqslant h_{1} v(u, u) \\
& +h_{2} v(u, F u)+h_{3} v(u, G u) \\
= & \left(h_{1}+h_{2}+h_{3}\right) v(u, u),
\end{aligned}
$$

which induces that $\left(e-h_{1}-h_{2}-h_{3}\right) v(u, u) \leqslant \theta$. Now, notice that $h_{1}+h_{2}+h_{3} \preccurlyeq s h_{1}+s h_{2}+s^{2} h_{3}$. Thus, by relation (4), $\left(e-h_{1}-h_{2}-h_{3}\right)$ is invertible. Hence, by Lemma $2,\left(l_{3}\right)$, we have $v(u, u)=\theta$. Next, we prove that the common fixed point of $F$ and $G$ is unique. Assume that $v$ is another common fixed point $F$ and $G$. It follows from relation (2) that

$$
\begin{aligned}
v(u, v)= & v(F u, G v) \leqslant h_{1} v(u, v) \\
& +h_{2} v(u, F u)+h_{3}(u, G v) \\
= & \left(h_{1}+h_{3}\right) v(u, v) .
\end{aligned}
$$

Since $h_{1}+h_{3} \preccurlyeq s h_{1}+s h_{2}+s^{2} h_{3}$ and by using relation (4), we have $v(u, v)=\theta$ by Lemma $2,\left(l_{3}\right)$. Also, it follows from relation (3) that

$$
\begin{aligned}
v(v, u)= & v(G v, F u) \leqslant h_{1} v(v, u) \\
& +h_{2} v(v, G v)+h_{3}(v, F u) \\
= & \left(h_{1}+h_{3}\right) v(v, u),
\end{aligned}
$$

which implies by the above procedure that $v(v, u)=\theta$. Now, by Lemma 6 , we obtain $u=v$. Consequently, the common fixed point of $F$ and $G$ is unique. Here, the proof ends.

Corollary 8. Consider a generalized c-distance $v$ on a complete cone b-metric space $(\mathfrak{X}, d)$ over a Banach algebra $\mathscr{E}$. Assume that two mappings $F, G: \mathfrak{X} \rightarrow \mathfrak{X}$ for every $a, b \in \mathfrak{X}$ satisfy the following relations: 


$$
\begin{aligned}
& v(F a, G b) \leqslant h_{1} v(a, b)+h_{2} v(a, F a), \\
& v(G a, F b) \leqslant h_{1} v(a, b)+h_{2} v(a, G a),
\end{aligned}
$$

where $h_{1}, h_{2} \in P$ with $\rho\left(s h_{1}+s h_{2}\right)<1$. Then, $F$ and $G$ have a unique common fixed point.

Proof. It is sufficient to set $h_{3}=\theta$ in Theorem 7 .

Corollary 9. Consider a generalized c-distance $v$ on a complete cone b-metric space $(\mathfrak{X}, d)$ over a Banach algebra $\mathscr{E}$. Assume that a mapping $F: \mathfrak{X} \rightarrow \mathfrak{X}$ for every $a, b \in \mathfrak{X}$ satisfies the following relation:

$$
v(F a, F b) \leqslant h_{1} v(a, b)+h_{2} v(a, F a),
$$

where $h_{1}, h_{2} \in P$ with $\rho\left(s h_{1}+s h_{2}\right)<1$. Then, $F$ has a unique fixed point.

Proof. It follows by taking $F=G$ in Corollary 8 .

Corollary 10. Consider a generalized c-distance $v$ on a complete cone b-metric space $(\mathfrak{X}, d)$ over a Banach algebra $\mathscr{E}$. Assume that a mapping $F: \mathfrak{X} \rightarrow \mathfrak{X}$ for every $a, b \in \mathfrak{X}$ satisfies the following relation:

$$
v(F a, F b) \leqslant h_{1} v(a, b),
$$
point.

where $h_{1} \in P$ with $\rho\left(h_{1}\right)<1 / s$. Then, $F$ has a unique fixed

Proof. It is sufficient to set $h_{2}=\theta$ in Corollary 9 .

Example 11. Let $\mathfrak{X}=[0,1], \mathscr{E}=C_{\mathbb{R}}^{1}[0,1]$ with the norm $\|f\|$ $=\|f\|_{\infty}+\left\|f^{\prime}\right\|_{\infty}$ and multiplication in $\mathscr{E}$ be just pointwise multiplication. Then, $\mathscr{E}$ is a real Banach algebra with a unit $e(t)=1$ for all $t \in[0,1]$. Take a solid cone $P=\{f \in \mathscr{E} \mid f(t)$ $\geq 0$ for all $t \in[0,1]\}$ and define the cone $b$-metric $d: \mathfrak{X} \times \mathfrak{X}$ $\rightarrow P \subseteq \mathscr{E}$ by $d(a, b)=|a-b|^{s} 2^{t}$, where $2^{t} \in P \subset \mathscr{E}$ and $s=2$. Consider a mapping $v: \mathfrak{X} \times \mathfrak{X} \rightarrow \mathscr{E}$ by $v(a, b)(t)=b^{2} 2^{t}$ for all $a, b, t \in \mathfrak{X}$. Then, $v$ is a generalized $c$-distance in cone $b$ -metric space $d$ over Banach algebra $\mathscr{E}$. Take $h_{1}=2 / 121+($ $3 / 121) t$ and define the mapping $F: \mathfrak{X} \rightarrow \mathfrak{X}$ by

$$
F(a)= \begin{cases}\frac{\sqrt{2}}{11} a, & a \in \mathbb{Q} \cap \mathfrak{X}, \\ \frac{\sqrt{2}}{12} a, & \text { otherwise. }\end{cases}
$$

Clearly, $F$ is not continuous. Also,

$$
\rho\left(s h_{1}\right)=\frac{10}{121}<1 .
$$

On the other hand, we have the following two cases: (i) for all $a \in \mathfrak{X}$ and $b \in \mathbb{Q} \cap \mathfrak{X}$, we get

$$
v(F a, F b)(t)=(F b)^{2} 2^{t}=\frac{2}{121} b^{2} 2^{t} \leqslant h_{1} v(a, b)(t)
$$

(ii) for all $a \in \mathfrak{X}$ and $b \in \mathbb{Q} \cap \mathfrak{X}$, we get

$$
v(F a, F b)(t)=(F b)^{2} 2^{t}=\frac{2}{144} b^{2} 2^{t} \leqslant h_{1} v(a, b)(t)
$$

That is, all hypotheses of Corollary 10 are held. Thus, $F$ has a unique fixed point at $a=0$.

Corollary 12. Consider a generalized c-distance $v$ on a complete cone $b$-metric space $(\mathfrak{X}, d)$. Assume that two mappings $F, G: \mathfrak{X} \rightarrow \mathfrak{X}$ for every $a, b \in \mathfrak{X}$ satisfy the following relations:

$$
\begin{aligned}
& v(F a, G b) \leqslant h_{1} v(a, b)+h_{2} v(a, F a)+h_{3} v(a, G b), \\
& v(G a, F b) \leqslant h_{1} v(a, b)+h_{2} v(a, G a)+h_{3} v(a, F b),
\end{aligned}
$$

where $h_{1}, h_{2}, h_{3} \in P$ so that $s\left(h_{1}+h_{2}\right)+\left(s^{2}+s\right) h_{3}<1$. Then, $F$ and $G$ have a unique common fixed point.

Proof. In Theorem 7, put $\rho\left(h_{1}\right)=h_{1}, \rho\left(h_{2}\right)=h_{2}$, and $\rho\left(h_{3}\right)$ $=h_{3}$. The proof is evident.

Remark 13. In Theorem 7 and its corollaries, we take $s=1$. Then, we obtain the same Theorem 16 and its next corollaries from Han and $\mathrm{Xu}[19]$ regarding a $c$-distance $v$ over a Banach algebra $\mathscr{E}$. Also, these results generalize some main theorems and its next corollaries in $[1,3,12,13,15,18,20]$.

\section{Conclusions}

In this paper, we established several fixed point results for two mappings $F$ and $G$ regarding a generalized $c$-distance $v$ over a Banach algebra $\mathscr{E}$. Notice that the class of these distances is bigger than the class of usual $c$-distances over the same Banach algebra. Also, this class is not equivalent to the class of $w t$-distances in $b$-metric spaces. Further, we removed the continuity condition of the mappings $F$ and $G$ in expressing our results.

\section{Data Availability}

No data were used to support this study.

\section{Conflicts of Interest}

The authors declare that they have no competing interests.

\section{Authors' Contributions}

All authors contributed equally and significantly in writing this article. All authors read and approved the final manuscript. 


\section{References}

[1] B. Bao, S. Xu, L. Shi, and V. Č. Rajć, "Fixed point theorems on generalized c-distance in ordered cone b-metric spaces," International Journal of Nonlinear Analysis and Applications, vol. 6, no. 1, pp. 9-22, 2015.

[2] I. A. Bakhtin, "The contraction mapping principle in quasimetric spaces," Functional Analysis, vol. 30, pp. 26-37, 1989.

[3] Y. J. Cho, R. Saadati, and S. Wang, "Common fixed point theorems on generalized distance in ordered cone metric spaces," Computers \& Mathematcs with Applications, vol. 61, no. 4, pp. 1254-1260, 2011.

[4] S. Czerwik, "Contraction mappings in b-metric spaces," Acta Mathematica et Informatica Universitatis Ostraviensis, vol. 1, pp. 5-11, 1993.

[5] M. M. Fréchet, "Sur quelques points du calcul fonctionnel," Rendiconti del Circolo Matematico di Palermo, vol. 22, no. 1, pp. 1-72, 1906.

[6] L. G. Huang and X. Zhang, "Cone metric spaces and fixed point theorems of contractive mappings," Journal of Mathematical Analysis and Applications, vol. 332, no. 2, pp. 14681476, 2007.

[7] N. Hussain, R. Saadati, and R. P. Agrawal, "On the topology and $w$-distance on metric type spaces," Fixed Point Theory and Applications, vol. 2014, no. 1, Article ID 88, 2014.

[8] N. Hussain and M. H. Shah, "KKM mappings in cone $b$-metric spaces," Computers \& Mathematcs with Applications, vol. 62, no. 4, pp. 1677-1684, 2011.

[9] O. Kada, T. Suzuki, and W. Takahashi, "Nonconvex minimization theorems and fixed point theorems in complete metric spaces," Mathematica Japonica, vol. 44, pp. 381-391, 1996.

[10] S. Wang and B. Guo, "Distance in cone metric spaces and common fixed point theorems," Applied Mathematics Letters, vol. 24, no. 10, pp. 1735-1739, 2011.

[11] L. Aryanpour, H. Rahimi, and G. Soleimani Rad, "Fixed point results for Hardy-Rogers type contractions with respect to a $\mathrm{c}$ -distance in graphical cone metric spaces," Issues of Analysis, vol. 27, no. 1, pp. 27-37, 2020.

[12] R. Babaei, H. Rahimi, M. De La Sen, and G. S. Rad, " $w$-b-cone distance and its related results: a survey," Symmetry, vol. 12, no. 1, p. 171, 2020.

[13] G. S. Rad, H. Rahimi, and C. Vetro, "Fixed point results under generalized c-distance with application to nonlinear fourthorder differential equation," Fixed Point Theory, vol. 20, no. 2, pp. 635-648, 2019.

[14] H. Liu and S. Xu, "Cone metric spaces with Banach algebras and fixed point theorems of generalized Lipschitz mappings," Fixed Point Theory and Applications, vol. 2013, no. 1, Article ID 320, 2013.

[15] A. Arabnia Firozjah, H. Rahimi, and G. Soleimani Rad, "Fixed and periodic point results in cone b-metric spaces over Banach algebras; a survey," Fixed Point Theory, vol. 22, no. 1, pp. 157168, 2021.

[16] H. Huang, G. Deng, and S. Radenović, "Some topological properties and fixed point results in cone metric spaces over Banach algebras," Positivity, vol. 23, no. 1, pp. 21-34, 2019.

[17] H. Huang, S. Hu, B. Z. Popović, and S. Radenović, "Common fixed point theorems for four mappings on cone $b$-metric spaces over Banach algebras," Journal of Nonlinear Sciences and Applications, vol. 9, no. 6, pp. 3655-3671, 2016.
[18] H. Huang, S. Radenović, and T. Dosenović, "Some common fixed point theorems on c-distance in cone metric spaces over Banach algebras," Applied and Computational Mathematics, vol. 14, no. 2, pp. 180-193, 2015.

[19] Y. Han and S. Xu, "Some new theorems on $c$-distance without continuity in cone metric spaces over Banach algebras," Journal of Function Spaces, vol. 2018, Article ID 7463435, 10 pages, 2018.

[20] A. Arabnia Firozjah, H. Rahimi, M. de la Sen, and G. Soleimani $\mathrm{Rad}$, "Fixed point results under generalized $c$-distance in cone $b$-metric spaces over Banach algebras," Axioms, vol. 9, no. 1, p. 31, 2020.

[21] W. Rudin, Functional Analysis, McGraw-Hill, New York, 1991. 